1

\title{
Plasma total-tau as a biomarker of stroke risk in the community
}

Matthew P. Pase (Ph.D.) ${ }^{1,2,3 \star}$, Jayandra J. Himali (Ph.D.) ${ }^{2,4,5 \star}$, Hugo J. Aparicio ${ }^{2,4}$

(MD, MPH), Jose Rafael Romero (MD) ${ }^{2}$, Claudia L. Satizabal (Ph.D. $)^{2,4,6}$, Pauline Maillard (Ph.D.) ${ }^{7}$, Charles DeCarli (MD) ${ }^{7}$, Alexa S. Beiser (Ph.D. $)^{2,4,5}$, Sudha

Seshadri (MD) $)^{2,6}$

*Joint first authors

${ }^{1}$ Melbourne Dementia Research Centre, The Florey Institute for Neuroscience and Mental Health \& The University of Melbourne, Melbourne, Australia

${ }^{2}$ Framingham Heart Study, Framingham, MA.

${ }^{3}$ Centre for Human Psychopharmacology, Swinburne University of Technology, Australia.

${ }^{4}$ Department of Neurology, Boston University School of Medicine, Boston, MA.

${ }^{5}$ Department of Biostatistics, Boston University School of Public Health, Boston, MA. ${ }^{6}$ Glenn Biggs Institute for Alzheimer's and Neurodegenerative Diseases, University of Texas Health Sciences Center, San Antonio, TX

${ }^{7}$ Department of Neurology, School of Medicine \& Imaging of Dementia and Aging Laboratory, Center for Neuroscience, University of California Davis, Sacramento CA.

Correspondence to: Dr. Pase. Melbourne Brain Centre. 30 Royal Parade, Parkville VIC, Australia, 3052. Phone: (+61)401267924. Email - matthewpase@gmail.com. Dr. Seshadri. 7703 Floyd Curl Drive, Mail Code 8070, University of Texas Health Sciences Center, San Antonio, TX 78229. Phone: 210-450-8437; email suseshad@bu.edu

This is the author manuscript accepted for publication and has undergone full peer review but has not been through the copyediting, typesetting, pagination and proofreading process, which may lead to differences between this version and the Version of Record. Please cite this article as doi: 10.1002/ana.25542

This article is protected by copyright. All rights reserved. 
Running title: Plasma tau and stroke risk

Title character count: 63 Running title character count: 26 Words count:

Abstract (=98), Introduction (=163), Discussion and manuscript body (=1499)

\begin{abstract}
Higher plasma total-tau level is associated with incident dementia but its relationship with stroke risk is unknown. In this prospective community-based study, we evaluated plasma total-tau level as a biomarker of stroke risk in 2794 Framingham Heart Study participants. Persons with plasma total-tau levels in the top quintile, versus the bottom four, had an increased risk of incident stroke over a mean followup of 8.3 years (Hazard Ratio, 2.01; 95\% Confidence Interval, 1.32-3.08) following adjustments for age, sex, and stroke risk factors. Our findings demonstrate that plasma total-tau relates to the risk of stroke in a community sample.
\end{abstract}

This article is protected by copyright. All rights reserved. 


\section{Introduction}

The tau protein is expressed in neurons and is involved in stabilizing microtubules and assisting with axonal maintenance and transport. ${ }^{1}$ Damage to neurons may cause tau to leak into the cerebrospinal fluid and blood, whereby elevated total-tau (t-tau) levels act as a non-specific marker of neuronal injury. ${ }^{2}$ We recently showed that higher plasma t-tau levels predicted the risk of incident dementia in the prospective, community-based Framingham Heart Study (FHS), with successful replication in the Memento Cohort. ${ }^{3}$ In that study, plasma t-tau was associated with the presence of both neurofibrillary tangles and microinfarcts at autopsy. We suspect that plasma t-tau levels may increase in response to brain injury from both vascular and neurodegenerative lesions predicting both stroke and dementia risk. Although we have demonstrated the latter, ${ }^{3}$ to our knowledge, plasma t-tau has not been examined with respect to the risk of incident stroke. Accordingly, we examined whether plasma t-tau associated with the risk of incident first-ever stroke in the community-based FHS.

\section{Methods}




\section{Study sample}

The FHS is an ongoing prospective community-based cohort in Massachusetts (USA). The study began in 1948 with the recruitment of an Original Cohort. Survivors have been followed prospectively with biennial examination cycles and ongoing surveillance for clinical events. In 1971, 5124 children of the Original cohort, as well as spouses of these children, were recruited into the Offspring Cohort. The Offspring Cohort has been studied across 9 examination cycles with detailed methods provided elsewhere. ${ }^{4}$ All participants provided written informed consent. The Institutional Review Board and Boston University Medical Center approved the study.

\section{Assessment of plasma t-tau}

Single Molecule Array (Simoa) technology permits the detection of t-tau from plasma with over 1000-fold improvement in sensitivity over conventional ELISA. ${ }^{5,6}$ At the eighth Offspring Cohort examination (2005-08), participants presented to the Heart Study clinic following an overnight fast. A blood draw was completed with samples immediately centrifuged, aliquoted and stored at $-80^{\circ} \mathrm{C}$. For this study, Quanterix (Lexington, MA) used these samples, which had never been thawed, to analyze plasma t-tau using a Simoa ${ }^{\text {TM }}$ Tau 2.0 Kit and a Simoa HD-1 analyzer. This singlemolecule digital ELISA is validated as fit-for-purpose research-use-only with a limit of detection at $0.019 \mathrm{pg} / \mathrm{mL}$. The assay detects all tau isoforms, including normal and 
phosphorylated tau. The analytical range was between 0.06 and $360 \mathrm{pg} / \mathrm{mL}$. The intra- and inter-assay coefficients of variation were $4.1 \%$ and $7.5 \%$, respectively.

\section{Stroke case ascertainment}

Stroke incidence was assessed through the continuous monitoring of hospital admissions in Framingham and by reviewing all available medical records and results. We also asked participants about the occurrence of stroke and stroke symptoms at FHS examination cycle and during annual FHS health status updates. Where possible, a study neurologist visited participants suspected of having a stroke within 48 hours. Stroke was defined as focal neurological symptoms of rapid onset and presumed vascular origin, lasting $>24$ hours or resulting in death within 24 hours. A committee comprising of least three FHS investigators, including at least two neurologists, adjudicated stroke diagnosis. The committee considered all available medical records, brain imaging, cerebrovascular imaging, and the assessment of the study neurologist who visited the participant.

\section{White Matter Hyperintensity Volume (WMHV)}

WMHV was estimated from structural brain MRI with a combination of FLAIR and 3D-T1 images. Values were expressed as a percentage of intracranial volume and log transformed. Full methods are provided elsewhere. ${ }^{7}$

\section{Statistical Methods}


Our analysis sample was derived from persons who attended the $8^{\text {th }}$ Offspring cohort examination cycle $(\mathrm{N}=3021)$ and had plasma t-tau measured $(\mathrm{N}=2885)$. We then excluded 13 participants aged under 45 years (since stroke in the young is rare), 71 persons with prevalent stroke, and 7 persons without stroke follow-up, leaving an analytical sample of 2794 .

We related plasma t-tau to the risk of incident stroke using Cox Proportional Hazards regression models. Follow-up was from the baseline exam to the time of incident event, death, or date the participant was last known to be stroke-free, through to 2016. We adjusted results according to two statistical models. Model 1 included adjustments for age and sex. Model 2 included the addition of clinical stroke risk factors: systolic blood pressure, treatment for hypertension, current smoking status, total cholesterol, high density lipoprotein cholesterol, prevalent atrial fibrillation (noted by a Framingham cardiologist on any ECG done at or before the baseline examination), diabetes, and prevalent cardiovascular disease (CVD; coronary heart disease, myocardial infarction, intermittent claudication, congestive heart failure). Next, we examined the usefulness of plasma t-tau to predict the risk of incident stroke over and above the Framingham Stroke Risk Profile (FSRP). The FSRP uses an updated algorithm that estimates the 10-year risk of stroke using age, sex, current smoking status, prevalent CVD, prevalent atrial fibrillation, diabetes, hypertension treatment, and systolic blood pressure. The FSRP was validated in three large community cohorts. ${ }^{8}$ We examined whether plasma t-tau could improve risk 
stratification for incident stroke over and the above the FSRP by calculating the continuous net reclassification improvement (NRI) applicable to survival data, taking into account events, non-events, and censoring. ${ }^{9,10}$ The $95 \%$ confidence interval $(\mathrm{Cl})$ was calculated using bootstrap methods. ${ }^{11}$ The continuous NRI quantifies upward and downward movement as any change in predicted probabilities, with higher values indicating superior discrimination. ${ }^{10}$ All analyses were conducted using SAS V9.4 (SAS Institute, Cary, N.C.). Missing data were excluded from analysis. Results were deemed significant if $\mathrm{P}<0.05$ (two-tailed).

\section{Results}

Cohort characteristics

Persons with high plasma t-tau were also more likely to have prevalent CVD, atrial fibrillation, and diabetes and were more likely to receive treatment for high blood pressure (Table 1).

\section{Plasma t-tau and the risk of incident stroke}

We observed $101(4 \%)$ cases of incident stroke over a mean follow-up of $8.3 \pm 2.1$ years, 82 were ischemic in origin. Plasma t-tau values were right skewed. We graphed the association between each quintile of plasma t-tau levels and the risk of incident stroke adjusting for age and sex (Fig 1a). From these data, we observed a threshold effect at the level of the top quintile. Thus, for all analyses, we created a binary variable and examined plasma t-tau values in the top quintile $(\geq 4.95 \mathrm{pg} / \mathrm{mL})$ 
versus the bottom 4. Persons with plasma t-tau in the top quintile displayed a greater than two-fold increase in the risk of stroke (Fig 1b, Table 2).

\section{Sensitivity analysis}

In a series of post-hoc sensitivity analyses (Table 2), we found similar effect sizes between plasma t-tau levels and stroke risk when excluding participants with prevalent dementia or other significant neurological diseases at baseline (e.g., multiple sclerosis), and when including additional adjustment for WMHV in a subsample with brain MRI. As compared with the primary analyses, we also found similar results when limiting the outcome to ischemic stroke, although attenuated slightly.

Plasma t-tau and the prediction of incident stroke Persons with plasma t-tau in the top quintile, relative to the remainder of the sample, had a 2.28-fold increase in the risk of stroke following adjustment for the FSRP (HR, 2.28; $95 \% \mathrm{Cl}, 1.51-3.44$ ). Adding plasma t-tau (at the threshold of the top quintile) to a model including the FSRP improved stroke risk discrimination (NRI overall, 0.47; 95\% Cl, 0.24-0.69; NRI events, -0.15; NRI non-events, 0.62).

\section{Discussion}


Our large study demonstrated that plasma t-tau associated with the risk of incident stroke in a community setting. A previous study of 50 acute ischemic stroke patients reported that plasma t-tau levels peaked 7 days post-stroke. ${ }^{12}$ Moreover, higher plasma t-tau was related to greater stroke severity, poorer stroke outcomes, and larger infarct volumes. Thus, levels of plasma t-tau may increase in response to vascular brain injury and other brain insults. We extend the present literature to show that higher t-tau levels associate with the risk of first-ever stroke in a community sample.

In the FHS and other population-based cohorts, a higher burden of subclinical cerebrovascular disease is associated with a higher risk of incident stroke. ${ }^{13,14}$ Thus, t-tau may predict the risk of incident stroke through associations with subclinical vascular brain injury. Although associations between plasma t-tau and stroke remained following adjustment for WMHV, this imaging marker does not capture the full spectrum of vascular brain injury. For example, we have reported previously that higher plasma t-tau levels predict a higher burden of microinfarcts at autopsy as well as lower hippocampal volumes on brain MRI. ${ }^{3}$ In this study, we were underpowered to examine other imaging markers of vascular brain injury since MRI was only available on a subset.

Our study is not without limitations. Our findings are based on only 101 incident stroke cases, and our sample was mostly of European descent, meaning that it is 
unclear how our data will generalize to other ethnic groups. Our results will require replication in independent cohorts with different population attributes. Our study was observational meaning that we could not infer a causal relationship between increased t-tau levels and incident stroke. As the t-tau assay is relatively new, the reproducibility of plasma t-tau within individuals and the comparability of values across different populations need further study. Future studies are needed to refine the thresholds most predictive of future stroke across populations and settings.

Acknowledgments - We thank the Framingham Heart Study participants for donating their time to our research. We thank Patrice Sutherland and Sue Blease of the Framingham Heart Study for their assistance. MPP is funded by a National Heart Foundation of Australia Future Leader Fellowship (102052). The Framingham Heart Study is supported by the National Heart, Lung, and Blood Institute (contract no. N01-HC-25195 and no. HHSN268201500001I) and by grants from the National Institute on Aging (R01 AG0059421, R01 AG054076, R01 AG049607, R01 AG058589, R01 AG059421, U01 AG049505, U01 AG052409) and the National Institute of Neurological Disorders and Stroke (NS017950 and UH2 NS100605). HJA is supported by a National Institute on Aging Research Supplement to Promote Diversity in Health-Related Research (R01AG054076-02S1) and through support from Boston University School of Medicine's Jack Spivack Neuroscience scholarship and Aram V. Chobanian Assistant Professorship. 
Author Contributions -Study concept and design: MPP, JJH, AB, SS; Acquisition, analysis, or interpretation of data: All authors. Drafting of the manuscript: MPP, JJH, $A B, S S$.

Potential Conflicts of Interest - Dr. DeCarli is a consultant to Novartis on a clinical trial of LCZ696 for heart failure.

\section{References}

1. Schraen-Maschke S, Sergeant N, Dhaenens C-M, et al. Tau as a biomarker of neurodegenerative diseases. Biomarkers in Medicine. 2008;2(4):363-384.

2. Jack CR, Jr., Bennett DA, Blennow K, et al. NIA-AA Research Framework: Toward a biological definition of Alzheimer's disease. Alzheimer's \& Dementia. 2018;14(4):535-562.

3. Pase MP, Beiser AS, Himali JJ, et al. Plasma total tau as a predictive biomarker for dementia and related endophenotypes. JAMA Neurology. 2019;76(5):598-606

4. Feinleib M, Kannel WB, Garrison RJ, McNamara PM, Castelli WP. The framingham offspring study. Design and preliminary data. Preventive Medicine. 1975;4(4):518-525.

5. Rissin DM, Kan CW, Campbell TG, et al. Single-molecule enzyme-linked immunosorbent assay detects serum proteins at subfemtomolar concentrations. Nature Biotechnology. 2010;28(6):595-599. 
6. Rissin DM, Fournier DR, Piech T, et al. Simultaneous Detection of Single Molecules and Singulated Ensembles of Molecules Enables Immunoassays with Broad Dynamic Range. Analytical Chemistry. 2011;83(6):2279-2285.

7. DeCarli C, Villeneuve S, Maillard P, et al. Vascular Burden Score Impacts Cognition Independent of Amyloid PET and MRI Measures of Alzheimer's Disease and Vascular Brain Injury. Journal of Alzheimer's disease. 2019;68(1):187-196.

8. Dufouil C, Beiser AS, McClure LA, et al. A Revised Framingham Stroke Risk Profile to Reflect Temporal Trends. Circulation. 2017;135(12):1145-1159.

9. Pencina MJ, D'Agostino RB, Sr., D'Agostino RB, Jr., Vasan RS. Evaluating the added predictive ability of a new marker: from area under the ROC curve to reclassification and beyond. Statistics in Medicine. 2008;27(2):157-172.

10. Pencina MJ, D'Agostino RB, Sr., Steyerberg EW. Extensions of net reclassification improvement calculations to measure usefulness of new biomarkers. Statistics in Medicine. 2011;30(1):11-21.

11. Hosmer DW, Lemeshow S. Confidence interval estimates of an index of quality performance based on logistic regression models. Statistics in Medicine. 1995;14(19):2161-2172.

12. De Vos A, Bjerke M, Brouns R, et al. Neurogranin and tau in cerebrospinal fluid and plasma of patients with acute ischemic stroke. BMC Neurology. 2017;17(1):170-170. 
13. Debette S, Beiser A, Decarli C, et al. Association of MRI markers of vascular brain injury with incident stroke, mild cognitive impairment, dementia, and mortality: The framingham offspring study. Stroke. 2010;41(4):600-606.

14. Wright CB, Dong C, Perez EJ, et al. Subclinical Cerebrovascular Disease Increases the Risk of Incident Stroke and Mortality: The Northern Manhattan Study. Journal of the American Heart Association. 2017;6(9).

\section{Figure legend.}

Figure 1. Risk of incident stroke for each quintile of plasma t-tau, relative to quintile one (A), and the adjusted cumulative incidence of stroke by quintile five of plasma ttau, versus quintiles 1-4 (B).

Legend: Data are for the incidence of all-cause stroke, adjusting for age and sex. The plasma t-tau values that defile in each quintile (in $\mathrm{pg} / \mathrm{mL}$ ) are; Q1, 0.12-3.02; Q2, >3.02-3.58; Q3, >3.58-4.14; Q4, >4.14-4.95; Q5, >4.95-106.75). The linear relationship between the log of plasma t-tau (per standard deviation unit increase) and the incidence of all-stroke was $\mathrm{HR}, 1.28(95 \% \mathrm{Cl}, 1.05,1.54)$ following 
adjustments for age and sex (Model 1) and $\mathrm{HR}, 1.22(95 \% \mathrm{Cl}, 1.01,1.49)$ following additional adjustments for stroke risk factors (Model 2). $\mathrm{Cl}=$ confidence interval, $\mathrm{HR}$ $=$ Hazard ratio, $\mathrm{Q}=$ quintile, $\mathrm{T}-\mathrm{Tau}=$ plasma total $=\mathrm{tau}$. 
A

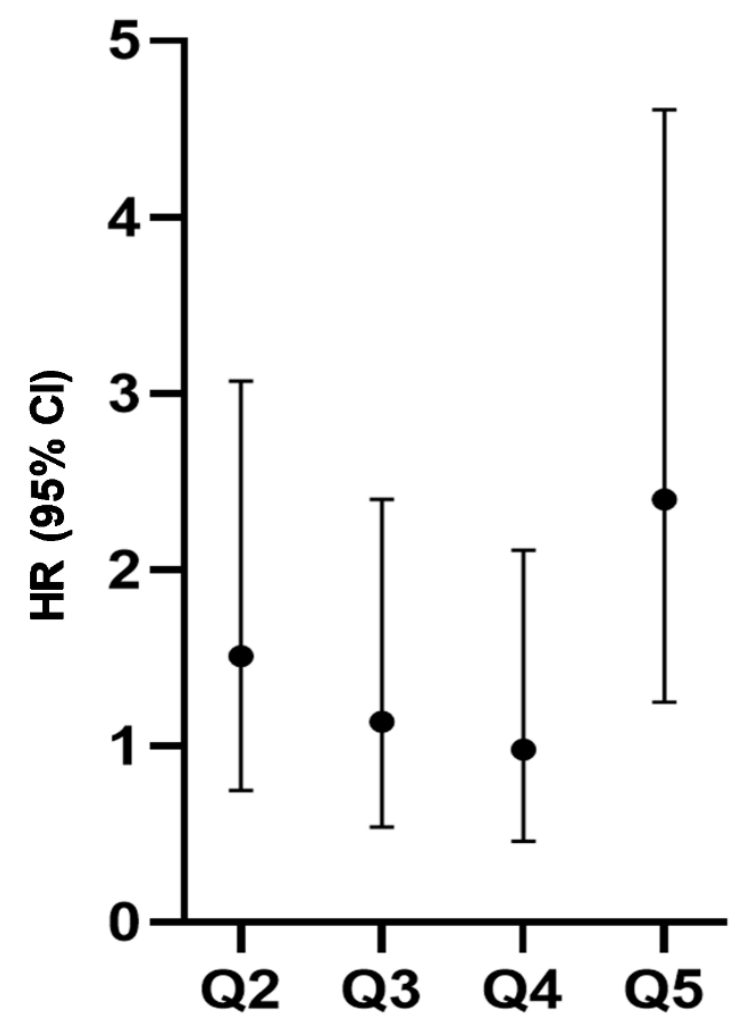

Quintile of T-Tau

(Relative to Quintile 1)

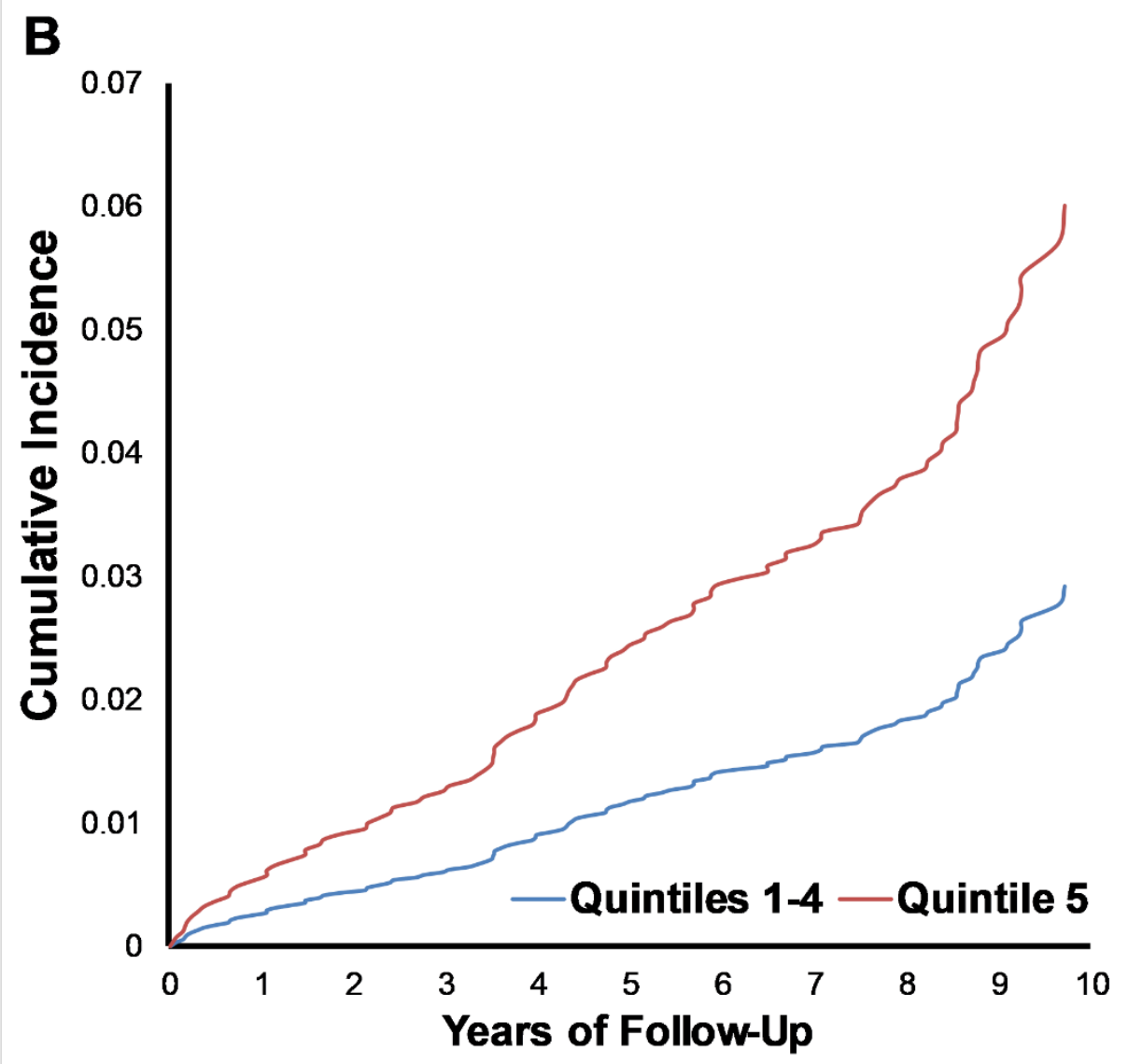

This article is protected by copyright. All rights reserved. 
This article is protected by copyright. All rights reserved. 
Table 1. Cohort characteristics

\begin{tabular}{lccc}
\hline & \multicolumn{2}{c}{ Plasma t-tau threshold } & \\
\cline { 2 - 3 } Characteristic & Q1-4 & Q5 & Overall \\
\hline No. of subjects & 2235 & 559 & 2794 \\
\hline Age, yr & $66 \pm 9$ & $70 \pm 9$ & $67 \pm 9$ \\
\hline Male, No. (\%) & $1094(49)$ & $179(32)$ & $1273(46)$ \\
\hline Total cholesterol, mg/dL & $187 \pm 37$ & $181 \pm 38$ & $186 \pm 37$ \\
\hline HDL cholesterol, mg/dL & $58 \pm 18$ & $57 \pm 18$ & $58 \pm 18$ \\
\hline Body mass index, kg/m ${ }^{2}$ & $28 \pm 5$ & $29 \pm 6$ & $28 \pm 5$ \\
\hline Current smoker, No. (\%) & $199(9)$ & $46(8)$ & $245(9)$ \\
\hline Systolic blood pressure, mmHg & $128 \pm 17$ & $131 \pm 19$ & $129 \pm 17$ \\
\hline Treatment for blood pressure, No. (\%) & $1124(50)$ & $364(65)$ & $1488(53)$ \\
\hline Prevalent diabetes mellitus, No. (\%) & $293(13)$ & $123(23)$ & $416(15)$ \\
\hline Prevalent CVD, No. (\%) & $268(12)$ & $130(23)$ & $398(14)$ \\
\hline APOE $\varepsilon 4$, No. (\%) & & $124(23)$ & $602(22)$ \\
\hline Prevalent atrial fibrillation, No. (\%) & $132(6)$ & $62(11)$ & $194(7)$ \\
\hline FSRP ${ }^{b}$, score units & $0.07 \pm 0.07$ & $0.1 \pm 0.1$ & $0.07 \pm 0.08$ \\
\hline Stroke follow-up, years & $8.4 \pm 2.0$ & $7.8 \pm 2.3$ & $8.3 \pm 2.1$ \\
\hline White matter hyperintensity volume, \% ${ }^{\mathrm{c}}$, & $0.17(0.10,0.34)$ & $0.24(0.11,0.46)$ & $0.18(0.10,0.36)$ \\
\hline median (Q1, Q3) & & & \\
\hline
\end{tabular}

CVD = cardiovascular disease; FSRP = Framingham Stroke Risk Profile; HDL = high-density lipoprotein; Q1, Q3 = quartile 1, quartile 3. The threshold for the plasma t-tau top quintile was $\geq 4.95 \mathrm{pg} / \mathrm{mL}$. Data are mean \pm standard deviation unless stated otherwise. ${ }^{\mathrm{a}}$ Positive for at least one APOE $\varepsilon 4$ allele. ${ }^{b}$ Estimates the 10 -year risk of stroke in persons free of stroke by assigning points based on a persons risk factor profile. ${ }^{\mathrm{C}}$ Available for a subset of 1581 persons with brain MRI. The overall median (quartile 1, quartile 3) level of plasma t-tau was $3.85 \mathrm{pg} / \mathrm{mL}(3.16,4.71)$.

This article is protected by copyright. All rights reserved. 
Table 2. Plasma t-tau and the risk of incident stroke

\begin{tabular}{|c|c|c|c|}
\hline \multirow[b]{2}{*}{$\begin{array}{l}\text { Model and plasma t- } \\
\text { tau criterion }\end{array}$} & \multicolumn{3}{|c|}{ Stroke Risk } \\
\hline & $\begin{array}{l}\text { No. of Events/ } \\
\text { No. at Risk }\end{array}$ & $\mathrm{HR}(95 \% \mathrm{Cl})$ & P Value \\
\hline \multicolumn{4}{|c|}{ Risk of all-stroke in Model $1^{a}$} \\
\hline Q5 ${ }^{b}$ & $40 / 559$ & $2.09(1.38,3.17)$ & 0.0005 \\
\hline Q1-4 & $61 / 2235$ & Reference & - \\
\hline \multicolumn{4}{|c|}{ Risk of all-stroke in Model $2^{c}$} \\
\hline Q5 ${ }^{b}$ & $40 / 541$ & $2.01(1.32,3.08)$ & 0.001 \\
\hline Q1-4 & $60 / 2170$ & Reference & - \\
\hline \multicolumn{4}{|l|}{ Sensitivity analysis $1^{d}$} \\
\hline Q5 ${ }^{b}$ & $31 / 553$ & $2.03(1.27,3.25)$ & 0.003 \\
\hline Q1-4 & $49 / 2211$ & Reference & - \\
\hline \multicolumn{4}{|l|}{ Sensitivity analysis $2^{\mathrm{e}}$} \\
\hline $\mathrm{Q}^{\mathrm{b}}$ & $39 / 545$ & $2.10(1.38,3.20)$ & 0.0006 \\
\hline Q1-4 & $59 / 2134$ & Reference & - \\
\hline \multicolumn{4}{|l|}{ Sensitivity analysis $3^{f}$} \\
\hline Q5 ${ }^{b}$ & $17 / 312$ & $2.00(1.08,3.71)$ & 0.03 \\
\hline Q1-4 & $30 / 1269$ & Reference & - \\
\hline
\end{tabular}

${ }^{\text {a }}$ Adjusts for age and sex.

${ }^{b}$ Threshold corresponds to the top quintile (Q5) with respect to the remainder of the sample (Q1-4).

${ }^{\mathrm{C}}$ Adjusts for age, sex, systolic blood pressure, treatment for hypertension, current smoking status, total cholesterol, High-density lipoprotein cholesterol, prevalent atrial fibrillation, diabetes, and cardiovascular disease.

${ }^{\mathrm{d}}$ Risk of ischemic stroke, adjusting for Model 1 covaraites.

${ }^{e}$ Risk of all-stroke excluding persons with prevalent dementia or other significant neurological disease at baseline, adjusting for Model 1 covariates.

${ }^{f}$ Risk of all-stroke after adjusting for white matter hyperintensity burden and Model 1 covariates. Based on a subsample of the larger cohort with brain MRI.

$\mathrm{Cl}=$ confidence interval, $\mathrm{HR}=$ hazard ratio, $\mathrm{Q}=$ quintile, $\mathrm{SD}=$ standard deviation. 


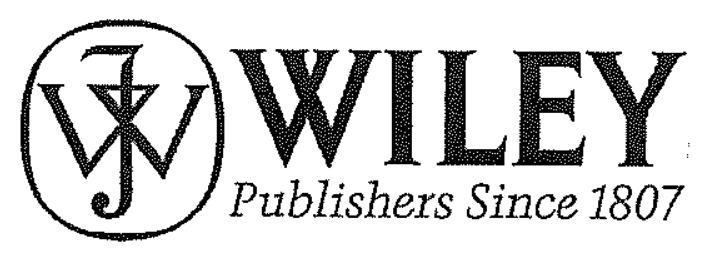

\section{COLOR REPRODUCTION IN YOUR ARTICLE}

These proofs have been typeset using the original figure files transmitted to production when this article was accepted for publication. Please review and mark your approval of each figure individually within your proof corrections. Should you need further assistance, please contact by e-mail dhineline@wiley.com

Because of the high cost of color printing we can only print figures in color if authors cover the expense. If you have submitted color figures please indicate your consent to cover the cost on the table listed below by marking the box corresponding to the approved cost on the table. The first color figure is $\$ 650$ USD and subsequent color figures are an additional $\$ 400$ USD.

Please note, all color images will be reproduced online at no charge, whether or not you opt for color printing. You will be invoiced for color charges once the article has been published in print.

Failure to return this form with your article proofs will delay the publication of your article.

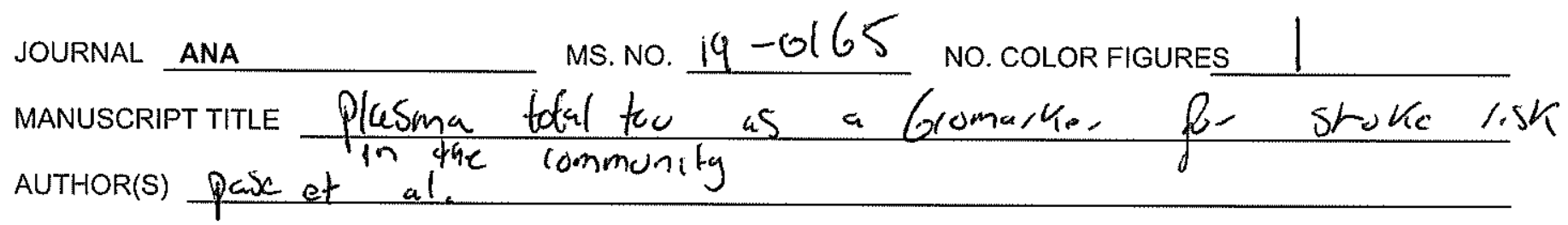

\begin{tabular}{|c|c|c|c|c|c|}
\hline No. Setor Figures & Color Charge & No. Color Figures & Color Charge & No. Color Figures & Color Charge \\
\hline \begin{tabular}{|l}
$\square$ \\
$\square$ \\
$\square$ \\
$\square$ \\
$\square$ \\
3 \\
4
\end{tabular} & $\begin{array}{l}\$ 650 \\
\$ 1050 \\
\$ 1450 \\
\$ 1850\end{array}$ & \begin{tabular}{|l}
$\square$ \\
$\square$ \\
$\square$ \\
$\square$ \\
7 \\
$\square$ \\
$\square$
\end{tabular} & $\begin{array}{l}\$ 2250 \\
\$ 2650 \\
\$ 3050 \\
\$ 3450\end{array}$ & 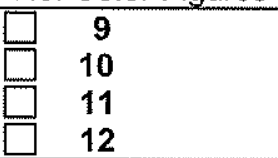 & $\begin{array}{l}\$ 3850 \\
\$ 4250 \\
\$ 4650 \\
\$ 5050\end{array}$ \\
\hline
\end{tabular}

Please print my figures color

Please print my figures in black and white

Please print the following figures in color

and convert these figures to black and white

Approved by Matthed Pase

Billing Address 30 Rogal Parade E-mail mpuse eunimell. edo.as Papkulk, vic, Austalic Telephone t61 401267924

Fax NA 


\section{Please wait...}

If this message is not eventually replaced by the proper contents of the document, your PDF viewer may not be able to display this type of document.

You can upgrade to the latest version of Adobe Reader for Windows®, Mac, or Linux® by visiting http://www.adobe.com/go/reader_download.

For more assistance with Adobe Reader visit http://www.adobe.com/go/acrreader.

Windows is either a registered trademark or a trademark of Microsoft Corporation in the United States and/or other countries. Mac is a trademark
of Apple Inc., registered in the United States and other countries. Linux is the registered trademark of Linus Torvalds in the U.S. and other countries. 


\section{Please wait...}

If this message is not eventually replaced by the proper contents of the document, your PDF viewer may not be able to display this type of document.

You can upgrade to the latest version of Adobe Reader for Windows®, Mac, or Linux® by visiting http://www.adobe.com/go/reader_download.

For more assistance with Adobe Reader visit http://www.adobe.com/go/acrreader.

Windows is either a registered trademark or a trademark of Microsoft Corporation in the United States and/or other countries. Mac is a trademark
of Apple Inc., registered in the United States and other countries. Linux is the registered trademark of Linus Torvalds in the U.S. and other countries. 


\section{Please wait...}

If this message is not eventually replaced by the proper contents of the document, your PDF viewer may not be able to display this type of document.

You can upgrade to the latest version of Adobe Reader for Windows®, Mac, or Linux® by visiting http://www.adobe.com/go/reader_download.

For more assistance with Adobe Reader visit http://www.adobe.com/go/acrreader.

Windows is either a registered trademark or a trademark of Microsoft Corporation in the United States and/or other countries. Mac is a trademark
of Apple Inc., registered in the United States and other countries. Linux is the registered trademark of Linus Torvalds in the U.S. and other countries. 


\section{Please wait...}

If this message is not eventually replaced by the proper contents of the document, your PDF viewer may not be able to display this type of document.

You can upgrade to the latest version of Adobe Reader for Windows®, Mac, or Linux® by visiting http://www.adobe.com/go/reader_download.

For more assistance with Adobe Reader visit http://www.adobe.com/go/acrreader.

Windows is either a registered trademark or a trademark of Microsoft Corporation in the United States and/or other countries. Mac is a trademark
of Apple Inc., registered in the United States and other countries. Linux is the registered trademark of Linus Torvalds in the U.S. and other countries. 
A

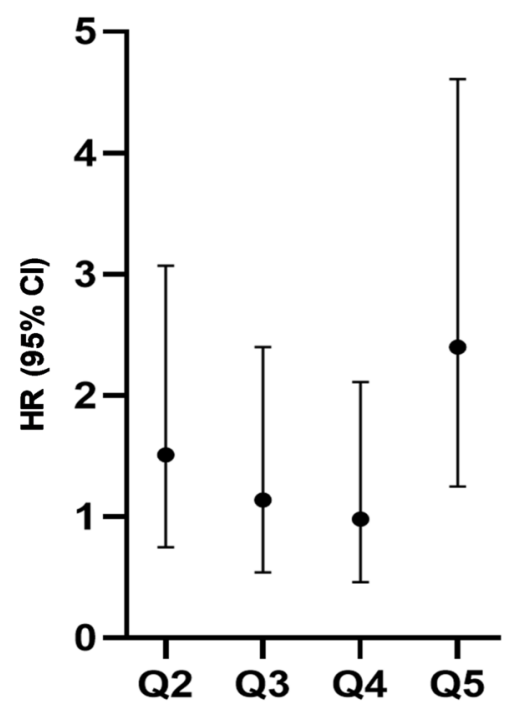

Quintile of T-Tau (Relative to Quintile 1)

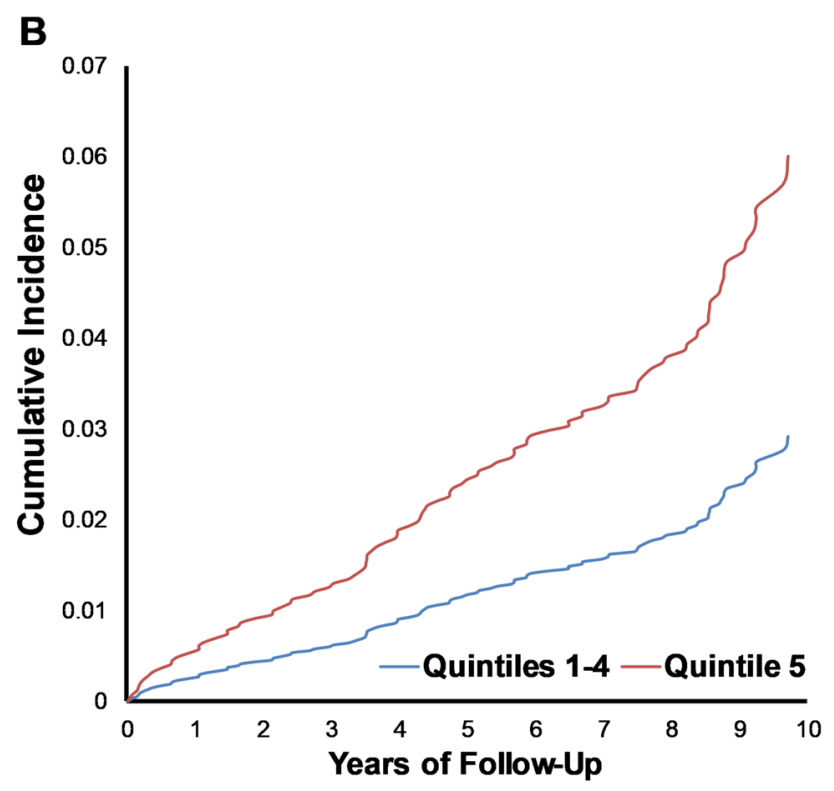

ANA_25542_Figure 1 A and B R2.tif

This article is protected by copyright. All rights reserved. 


\section{Please wait...}

If this message is not eventually replaced by the proper contents of the document, your PDF viewer may not be able to display this type of document.

You can upgrade to the latest version of Adobe Reader for Windows®, Mac, or Linux® by visiting http://www.adobe.com/go/reader_download.

For more assistance with Adobe Reader visit http://www.adobe.com/go/acrreader.

Windows is either a registered trademark or a trademark of Microsoft Corporation in the United States and/or other countries. Mac is a trademark
of Apple Inc., registered in the United States and other countries. Linux is the registered trademark of Linus Torvalds in the U.S. and other countries. 


\section{Please wait...}

If this message is not eventually replaced by the proper contents of the document, your PDF viewer may not be able to display this type of document.

You can upgrade to the latest version of Adobe Reader for Windows®, Mac, or Linux® by visiting http://www.adobe.com/go/reader_download.

For more assistance with Adobe Reader visit http://www.adobe.com/go/acrreader.

Windows is either a registered trademark or a trademark of Microsoft Corporation in the United States and/or other countries. Mac is a trademark
of Apple Inc., registered in the United States and other countries. Linux is the registered trademark of Linus Torvalds in the U.S. and other countries. 


\section{Please wait...}

If this message is not eventually replaced by the proper contents of the document, your PDF viewer may not be able to display this type of document.

You can upgrade to the latest version of Adobe Reader for Windows®, Mac, or Linux® by visiting http://www.adobe.com/go/reader_download.

For more assistance with Adobe Reader visit http://www.adobe.com/go/acrreader.

Windows is either a registered trademark or a trademark of Microsoft Corporation in the United States and/or other countries. Mac is a trademark
of Apple Inc., registered in the United States and other countries. Linux is the registered trademark of Linus Torvalds in the U.S. and other countries. 


\section{Please wait...}

If this message is not eventually replaced by the proper contents of the document, your PDF viewer may not be able to display this type of document.

You can upgrade to the latest version of Adobe Reader for Windows®, Mac, or Linux® by visiting http://www.adobe.com/go/reader_download.

For more assistance with Adobe Reader visit http://www.adobe.com/go/acrreader.

Windows is either a registered trademark or a trademark of Microsoft Corporation in the United States and/or other countries. Mac is a trademark
of Apple Inc., registered in the United States and other countries. Linux is the registered trademark of Linus Torvalds in the U.S. and other countries. 


\section{Please wait...}

If this message is not eventually replaced by the proper contents of the document, your PDF viewer may not be able to display this type of document.

You can upgrade to the latest version of Adobe Reader for Windows®, Mac, or Linux® by visiting http://www.adobe.com/go/reader_download.

For more assistance with Adobe Reader visit http://www.adobe.com/go/acrreader.

Windows is either a registered trademark or a trademark of Microsoft Corporation in the United States and/or other countries. Mac is a trademark
of Apple Inc., registered in the United States and other countries. Linux is the registered trademark of Linus Torvalds in the U.S. and other countries. 
Table 1. Cohort characteristics

\begin{tabular}{|c|c|c|c|}
\hline \multirow[b]{2}{*}{ Characteristic } & \multicolumn{2}{|c|}{ Plasma t-tau threshold } & \multirow[b]{2}{*}{ Overall } \\
\hline & Q1-4 & Q5 & \\
\hline No. of subjects & 2235 & 559 & 2794 \\
\hline Age, yr & $66 \pm 9$ & $70 \pm 9$ & $67 \pm 9$ \\
\hline Male, No. (\%) & $1094(49)$ & $179(32)$ & $1273(46)$ \\
\hline Total cholesterol, mg/dL & $187 \pm 37$ & $181 \pm 38$ & $186 \pm 37$ \\
\hline HDL cholesterol, mg/dL & $58 \pm 18$ & $57 \pm 18$ & $58 \pm 18$ \\
\hline Body mass index, $\mathrm{kg} / \mathrm{m}^{2}$ & $28 \pm 5$ & $29 \pm 6$ & $28 \pm 5$ \\
\hline Current smoker, No. (\%) & $199(9)$ & $46(8)$ & $245(9)$ \\
\hline Systolic blood pressure, $\mathrm{mmHg}$ & $128 \pm 17$ & $131 \pm 19$ & $129 \pm 17$ \\
\hline Treatment for blood pressure, No. (\%) & $1124(50)$ & $364(65)$ & 1488(53) \\
\hline Prevalent diabetes mellitus, No. (\%) & $293(13)$ & $123(23)$ & $416(15)$ \\
\hline Prevalent CVD, No. (\%) & $268(12)$ & $130(23)$ & $398(14)$ \\
\hline APOE $\varepsilon 4$, No. $(\%)^{a}$ & $478(22)$ & $124(23)$ & $602(22)$ \\
\hline Prevalent atrial fibrillation, No. (\%) & $132(6)$ & $62(11)$ & $194(7)$ \\
\hline FSRP $^{b}$, score units & $0.07 \pm 0.07$ & $0.1 \pm 0.1$ & $0.07 \pm 0.08$ \\
\hline Stroke follow-up, years & $8.4 \pm 2.0$ & $7.8 \pm 2.3$ & $8.3 \pm 2.1$ \\
\hline $\begin{array}{l}\text { White matter hyperintensity volume, } \%^{\mathrm{C}} \text {, } \\
\text { median (Q1, Q3) }\end{array}$ & $0.17(0.10,0.34)$ & $0.24(0.11,0.46)$ & $0.18(0.10,0.36)$ \\
\hline
\end{tabular}

CVD = cardiovascular disease; FSRP = Framingham Stroke Risk Profile; HDL = high-density lipoprotein; Q1, Q3 = quartile 1, quartile 3. The threshold for the plasma t-tau top quintile was $\geq 4.95 \mathrm{pg} / \mathrm{mL}$. Data are mean \pm standard deviation unless stated otherwise. ${ }^{\mathrm{a}}$ Positive for at least one APOE $\varepsilon 4$ allele. ${ }^{b}$ Estimates the 10 -year risk of stroke in persons free of stroke by assigning points based on a persons risk factor profile. ${ }^{\mathrm{C}}$ Available for a subset of 1581 persons with brain MRI. The overall median (quartile 1, quartile 3) level of plasma t-tau was $3.85 \mathrm{pg} / \mathrm{mL}(3.16,4.71)$.

This article is protected by copyright. All rights reserved. 
Table 2. Plasma t-tau and the risk of incident stroke

\begin{tabular}{lccc}
\hline & \multicolumn{3}{c}{ Stroke Risk } \\
\hline Model and plasma t- \\
\cline { 2 - 3 } tau criterion & No. of Events/ & HR $(95 \% \mathrm{Cl})$ & P Value \\
& No. at Risk & &
\end{tabular}

\begin{tabular}{cccc}
\hline \multicolumn{2}{l}{ Risk of all-stroke in Model $\mathbf{1}^{\mathbf{a}}$} & & \\
\hline Q5 $^{\mathbf{b}}$ & $40 / 559$ & $2.09(1.38,3.17)$ & 0.0005 \\
\hline Q1-4 & $61 / 2235$ & Reference & -
\end{tabular}

Risk of all-stroke in Model $2^{\mathrm{c}}$

$\begin{array}{lll}\text { Q5 }^{\text {b }} \quad 40 / 541 & 2.01(1.32,3.08) & 0.001\end{array}$

Q1-4 60/2170 Reference -

Sensitivity analysis $1^{d}$

$\begin{array}{cccc}\text { Q5 }^{\text {b }} & 31 / 553 & 2.03(1.27,3.25) & 0.003 \\ \text { Q1-4 } & 49 / 2211 & \text { Reference } & -\end{array}$

Sensitivity analysis $2^{\mathrm{e}}$

\begin{tabular}{cccc}
\hline Q5 $^{\text {b }}$ & $39 / 545$ & $2.10(1.38,3.20)$ & 0.0006 \\
\hline Q1-4 & $59 / 2134$ & Reference & - \\
\hline Sensitivity analysis 3 $^{f}$ & & & \\
\hline Q5 $^{\text {b }}$ & $17 / 312$ & $2.00(1.08,3.71)$ & 0.03 \\
\hline Q1-4 & $30 / 1269$ & Reference & -
\end{tabular}

${ }^{a}$ Adjusts for age and sex.

${ }^{\mathrm{b}}$ Threshold corresponds to the top quintile (Q5) with respect to the remainder of the sample (Q1-4).

${ }^{c}$ Adjusts for age, sex, systolic blood pressure, treatment for hypertension, current smoking status, total cholesterol, High-density lipoprotein cholesterol, prevalent atrial fibrillation, diabetes, and cardiovascular disease.

${ }^{\mathrm{d}}$ Risk of ischemic stroke, adjusting for Model 1 covaraites.

${ }^{\mathrm{e}}$ Risk of all-stroke excluding persons with prevalent dementia or other significant neurological disease at baseline, adjusting for Model 1 covariates.

${ }^{f}$ Risk of all-stroke after adjusting for white matter hyperintensity burden and Model 1 covariates. Based on a subsample of the larger cohort with brain MRI.

$\mathrm{Cl}=$ confidence interval, $\mathrm{HR}=$ hazard ratio, $\mathrm{Q}=$ quintile, $\mathrm{SD}=$ standard deviation.

This article is protected by copyright. All rights reserved. 


\section{University Library}

\section{- M M N E R VA A gateway to Melbourne's research publications}

Minerva Access is the Institutional Repository of The University of Melbourne

Author/s:

Pase, MP;Himali, JJ;Aparicio, HJ;Romero, JR;Satizabal, CL;Maillard, P;DeCarli, C;Beiser, AS;Seshadri, S

Title:

Plasma total-tau as a biomarker of stroke risk in the community

Date:

2019-07-29

\section{Citation:}

Pase, M. P., Himali, J. J., Aparicio, H. J., Romero, J. R., Satizabal, C. L., Maillard, P., DeCarli, C., Beiser, A. S. \& Seshadri, S. (2019). Plasma total-tau as a biomarker of stroke risk in the community. ANNALS OF NEUROLOGY, 86 (3), pp.463-467. https://doi.org/10.1002/ ana.25542.

Persistent Link:

http://hdl.handle.net/11343/286215 